\title{
Takagi-Sugeno Fuzzy Controller and Sliding Mode Controller for a Nonholonomic Mobile Robot
}

\author{
Hafedh Abid (i) \\ Laboratory of Sciences and Techniques of Automatic Control and Computer Engineering (Lab-STA) Sfax, \\ National School of Engineering of Sfax, University of Sfax, Sfax, Tunisia \\ Correspondence should be addressed to Hafedh Abid; abidhafedh@gmail.com
}

Received 16 April 2021; Revised 18 May 2021; Accepted 8 June 2021; Published 18 June 2021

Academic Editor: Zain Anwar Ali

Copyright ( 92021 Hafedh Abid. This is an open access article distributed under the Creative Commons Attribution License, which permits unrestricted use, distribution, and reproduction in any medium, provided the original work is properly cited.

This paper focuses on the nonholonomic wheeled mobile robot. We have presented a scheme to develop controllers. Two controllers have been developed. The first concerns the kinematic behavior, while the second relates to the dynamic behavior of the mobile robot. For the kinematic controller, we have used a Takagi-Sugeno fuzzy system to overcome the nonlinearities present in model, whereas for the second controller, we have used the sliding mode approach. The sliding surface has the identical structure as the proportional integral controller. The stability of the system has been proved based on the Lyapunov approach. The simulation results show the efficiency of the proposed control laws.

\section{Introduction}

In the last decades, the path of travel is considered as one of the critical problems in the field of mobile robotics. The trajectory tracking consists of guiding the robot through intermediate points to reach the final destination. This tracking is carried out under a constraint time, which means that the robot must reach the goal within a predefined time. In the literature, the problem is treated as the tracking of a reference robot that moves to the desired trajectory with a certain rhythm. The real robot must follow precisely the reference and reduce the distance error, by varying its linear and angular velocities $[1,2]$. There are many works that have focused on tracking the trajectory of the mobile robots, and they consider the mobile robot as a particle; in this case, the inputs are velocities. Their aims are kinematic models. In [3], the kinematic control law approach supposes that the control signal generates the exact motion commanded. On the contrary, some works consider the kinematic aspect and the dynamic aspect for the mobile robot. In this case, the actuator inputs signals are torques instead of velocities [4]. In [5], Lee et al. suggest a technique for designing the tracking control of wheeled mobile robots based on a new sliding surface with an approach angle. In [6], authors proposed a robust backstepping controller for the uncertain kinematic model of the wheeled mobile robot based on a nonlinear disturbance observer in order to cope with model uncertainties and the external disturbances. Topalov [7] proposed an adaptive fuzzy approach for the kinematic controller. This method was able to decrease the effect of unmodeled disturbances. In [8], a dynamic Petri recurrent fuzzy neural network was proposed. In [9], the proposed controller combines nonlinear time varying feedback with an integral sliding mode controller. The latter is obtained by introducing an integral term in the switching manifold.

In [10], a robust adaptive mobile robot controller is presented using backstepping for kinematics and dynamics motions, and the adaptive process was based on the neural network. In [11], a classical parallel distributed compensation (PDC) control law, based on Takagi-Sugeno fuzzy modeling, is proposed. The controller comprises sixteen rules in which the control gains have been calculated using LMI techniques. In [12], the authors present an adaptive controller with consideration of unknown model parameters.

In [13], the authors suggest a controller of a mobile robot in Cartesian coordinates with an approach angle based on the sliding mode. In [14], the authors combine hybrid backstepping kinematic control with the adaptive integral 
sliding mode kinetic control of the three-wheeled mobile robot.

Most of the works deal with nonholonomic wheeled mobile robot, which is used for kinematic motion of a classical controller arising from the backstepping method $[2,10,12,14,15]$.

This paper includes two main contributions. First, a new controller based on Takagi-Sugeno fuzzy systems for kinematic motion. This lastly uses three fuzzy rules. The second contribution consists of developing for the dynamic part a controller based on the sliding mode. The sliding surface, which is based on linear and angular velocities of the robot, has the similar structure as the proportional integral controller. The switching control term of the latter controller combines the two sliding surfaces.

The remainder of this paper is organized as follows. Section 2 is devoted to the description of the kinematic and dynamic models of the two-wheeled mobile robot. Section 3 that is reserved to the controllers design includes two subsections, the first is reserved to the development of the new T-S type fuzzy controller of the kinematic behavior, whereas the second is consecrated to the design of the dynamic motion controller using the sliding mode approach. The stability analysis is checked in the both precedent subsections by the Lyapunov approach. Then, Section 4 is sacred to the presentation of the simulation results.

\section{Mobile Robot Modeling}

In this section, we are interested in the modeling of the robot, which is composed of two driving wheels and a drive shaft in the center, as shown in Figure 1. Indeed, the Section 2.1 is reserved for kinematic modeling, while Section 2.2 concerns dynamic modeling.

We define the current position $\left(x_{c}, y_{c}\right)$ and the heading angle $\theta$, which constitute the coordinates of the middle point of the mobile robot and the angle between the heading direction and the $x$-axis to describe the current posture position of the mobile robot. Figure 1 depicts the current posture position of a two wheels mobile robot in Cartesian frame coordinates.

The nonholonomic constraint of a wheeled mobile robot is given by the following equation:

$$
\dot{y} \cos \theta-\dot{x} \sin \theta=0 \text {. }
$$

2.1. Fuzzy Kinematic Model of Robot. Based on the Newton-Euler equations [16] and the previous hypotheses, the state equations of the mobile robot are represented by the following equations' system [17]:

$$
\begin{aligned}
& \left\{\begin{array}{l}
\dot{x}=v \cos \theta, \\
\dot{y}=v \sin \theta, \\
\dot{\theta}=\omega,
\end{array}\right. \\
& v=\sqrt{\dot{x}^{2}+\dot{y}^{2},}
\end{aligned}
$$

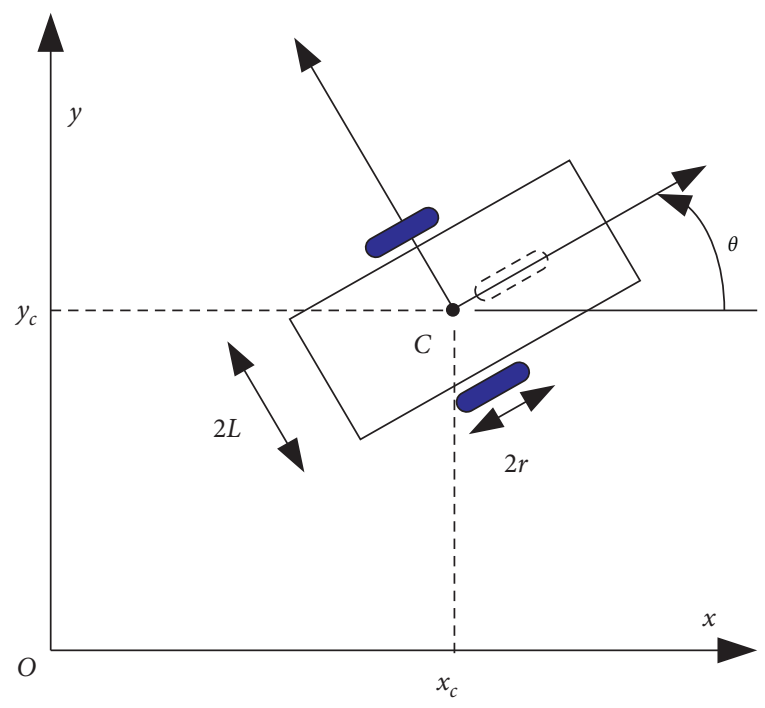

FIGURE 1: Representation of the navigation environment.

where $(x, y), v$, and $\omega$ represent, respectively, the instantaneous position coordinates of point $\mathrm{C}$ of the mobile robot in the global Cartesian frame and the measurements at point $\mathrm{C}$ of the linear and angular speeds of the robot. The state variables of mobile robot are $q=\left[\begin{array}{lll}x & y & \theta\end{array}\right]^{T}$ :

$$
\begin{aligned}
& v_{d}=\sqrt{\dot{x}_{d}^{2}+\dot{y}_{d}^{2},} \\
& w_{d}=\frac{\dot{x}_{d} \ddot{y}_{d}-\dot{y}_{d} \ddot{x}_{d}}{\dot{x}_{d}^{2}+\dot{y}_{d}^{2}}
\end{aligned}
$$

where $v_{d}$ and $w_{d}$ represent, respectively, the desired linear and angular velocity.

The state kinematic model of the mobile robot in Cartesian frame coordinates is given by the following expression:

$$
\dot{q}=\left[\begin{array}{c}
\dot{x} \\
\dot{y} \\
\dot{\theta}
\end{array}\right]=\left[\begin{array}{cc}
\cos \theta & 0 \\
\sin \theta & 0 \\
0 & 1
\end{array}\right]\left[\begin{array}{c}
v(t) \\
w(t)
\end{array}\right]=J(\theta) V_{m},
$$

with

$$
\begin{aligned}
V_{m} & =\left[\begin{array}{ll}
v & \omega
\end{array}\right]^{T}, \\
J(\theta) & =\left[\begin{array}{ccc}
\cos \theta & \sin \theta & 0 \\
0 & 0 & 1
\end{array}\right]^{T} .
\end{aligned}
$$

In order to develop a T-S fuzzy controller, which stabilizes the system and allows the robot to follow the desired path, we need a fuzzy model. In this context, we proceed to determine a fuzzy model of the robot.

The posture vector error is not specified in the global frame coordinate system, but quite as a vector error in the local frame coordinate system of the robot: $q_{e}(t)=$ $\left[\begin{array}{lll}e_{1} & e_{2} & e_{3}\end{array}\right]^{T}$.

The posture vector error $q_{e}$ is computed based on the actual posture vector $q(t)=\left[\begin{array}{lll}x & y & \theta\end{array}\right]^{T}$ and the reference posture vector $q_{d}(t)=\left[\begin{array}{lll}x_{d} & y_{d} & \theta_{d}\end{array}\right]^{T}$ : 


$$
\dot{q}_{d}(t)=J\left(\theta_{d}\right) V_{m d},
$$

where $V_{m d}=\left[\begin{array}{ll}v_{d} & \omega_{d}\end{array}\right]^{T}$.

So,

$$
\tilde{q}=q_{d}-q=\left[\begin{array}{l}
x_{d}-x \\
y_{d}-y \\
\theta_{d}-\theta
\end{array}\right]=\left[\begin{array}{l}
e_{x} \\
e_{y} \\
e_{\theta}
\end{array}\right] .
$$

The relation between the local frame and the global frame, as shown in Figure 2, is given by the following equation:

$$
q_{e}=\Re_{e} \widetilde{q}
$$

where

$$
\mathfrak{R}_{e}=\left[\begin{array}{ccc}
\cos \theta & \sin \theta & 0 \\
-\sin \theta & \cos \theta & 0 \\
0 & 0 & 1
\end{array}\right] \text {. }
$$

Equation (8) allows transforming the magnitudes described in the global coordinate system to the local coordinate system:

$$
\dot{q}_{e}=\dot{\mathfrak{R}}_{e} \widetilde{q}+\Re_{e} \dot{\tilde{q}}
$$

However, by differentiating equation (10), which contains the linear speed and the angular speed terms, we obtain the derivative of the error vector, which is expressed by the following equation:

$$
\dot{q}_{e}=\left\{\begin{array}{l}
\dot{e}_{1}=\omega e_{2}-v+v_{d} \cos e_{3}, \\
\dot{e}_{2}=-\omega e_{1}+v_{d} \sin e_{3}, \\
\dot{e}_{3}=\omega_{d}-\omega .
\end{array}\right.
$$

The posture error model can be rewritten as follows:

$$
\left[\begin{array}{l}
\dot{e}_{1} \\
\dot{e}_{2} \\
\dot{e}_{3}
\end{array}\right]=\left[\begin{array}{cc}
\cos e_{3} & 0 \\
\sin e_{3} & 0 \\
0 & 1
\end{array}\right]\left[\begin{array}{c}
v_{d} \\
w_{d}
\end{array}\right]+\left[\begin{array}{cc}
-1 & e_{2} \\
0 & -e_{1} \\
0 & 1
\end{array}\right]\left[\begin{array}{l}
v \\
w
\end{array}\right] .
$$

We note that equation (12) contains trigonometric nonlinearities which are $\cos \left(e_{3}\right)$ and $\sin \left(e_{3}\right)$. However, the nonlinearities depend on the error $e_{3}$, whose range of variation is from $-\mathrm{pi} / 2$ to $\mathrm{pi} / 2$.

The advantage of the T-S type fuzzy approach is that it allows describing the nonlinear model by linear submodels. Indeed, each submodel represents a local linear relation between the inputs and the outputs and all the nonlinearities are reported in the premises of the fuzzy rules [18].

Based on the theory of T-S fuzzy systems, the nonlinear model (12) can be transformed into three local models, which are inferred by fuzzy rules. The three local models are described by the following systems of equations:

From the weights assigned to each rule, the state vector of the fuzzy models is inferred as follows (which corresponds to a barycentric aggregation).

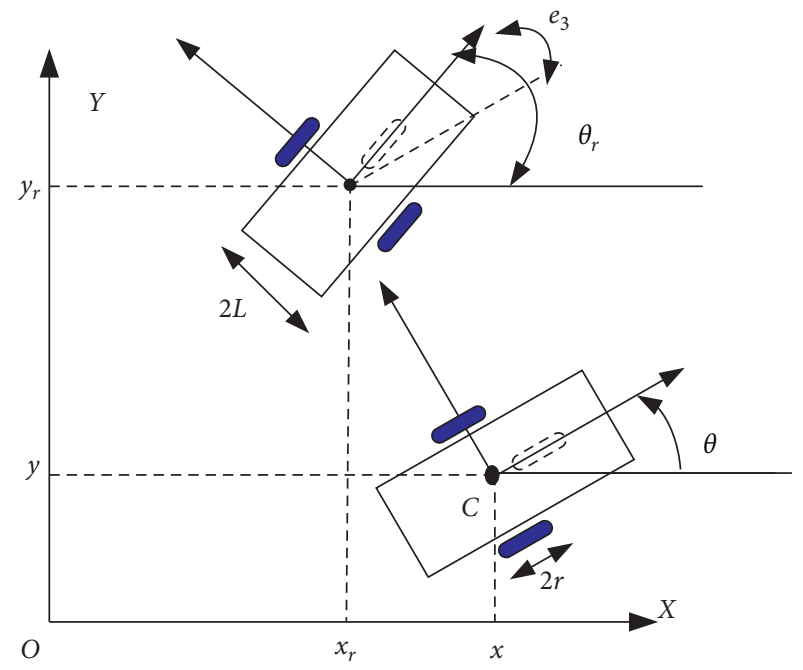

FIGURE 2: Trajectory tracking.

The member ship function for the error $e_{3}$ is given in Figure 3.

The rules of the local models are given by the following expression:

$$
\begin{aligned}
& \text { if } e_{3} \text { is } \mu_{1}, \\
& \text { if } e_{3} \text { is } \mu_{2}, \\
& \text { if } e_{3} \text { is } \mu_{3},
\end{aligned}
$$

The T-S fuzzy model of equation (12) is given by the following equation:

$$
\dot{q}_{e}=\sum_{i=1}^{3} \mu_{i}\left(A_{i} V_{m d}+B_{i} V_{m}\right)
$$

where $\mu_{i}$ and $A_{i}$ and $B_{i}$ represent, respectively, the weight assigned to each rule and the matrices associated to the local model.

With,

$$
\begin{aligned}
& A_{1}=\left[\begin{array}{ll}
1 & 0 \\
e_{3} & 0 \\
0 & 1
\end{array}\right], \\
& A_{2}=\left[\begin{array}{ll}
0 & 0 \\
1 & 0 \\
0 & 1
\end{array}\right], \\
& A_{3}=\left[\begin{array}{ll}
0 & 0 \\
-1 & 0 \\
0 & 1
\end{array}\right], \\
& B_{1}=B_{2}=B_{3}=\left[\begin{array}{cc}
-1 & e_{2} \\
0 & -e_{1} \\
0 & 1
\end{array}\right] .
\end{aligned}
$$




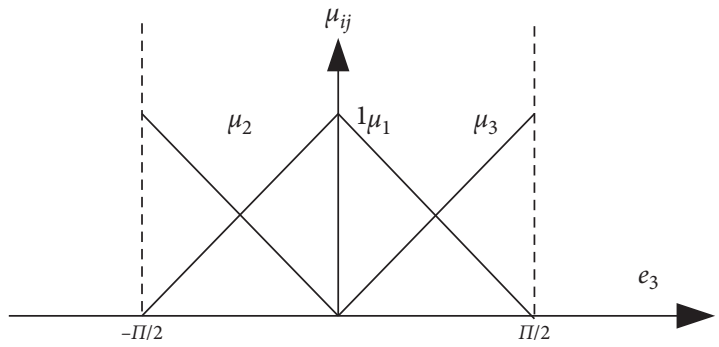

FIgURE 3: Membership function.

2.2. Dynamic Model of Robot. The dynamic equation of the wheeled mobile robot is given by the following equation:

$$
M(q) \ddot{q}+C(q, \dot{q}) \dot{q}+F(\dot{q})=B(q) \tau-A^{T}(q) \lambda,
$$

where $C(q, \dot{q})$ is the centripetal and Coriolis matrix, $F(\dot{q})$ is the friction force, $\tau$ represents the torque vector, and $A^{T}(q)=0$ :

$$
\begin{gathered}
M(q)=\left[\begin{array}{lll}
m & 0 & 0 \\
0 & m & 0 \\
0 & 0 & J_{g}
\end{array}\right], \\
B(q)=\frac{1}{r}\left[\begin{array}{cc}
\cos \theta & \cos \theta \\
\sin \theta & \sin \theta \\
L & -L
\end{array}\right], \\
C(q, \dot{q})=\left[\begin{array}{lll}
0 & 0 & 0 \\
0 & 0 & 0 \\
0 & 0 & 0
\end{array}\right],
\end{gathered}
$$

where $m$ and $J_{g}$ represent, respectively, the mass and the moment inertia of the wheeled mobile robot. $L$ and $r$ represent, respectively, the distance separating the two driving wheels and the wheel radius. Without considering disturbances and uncertainties, the latest equation becomes as

$$
\bar{M}(q) \dot{V}_{m}=\bar{B}(q) \tau,
$$

where

$$
\begin{aligned}
\bar{M}(q) & =\left[\begin{array}{ll}
m & 0 \\
0 & J_{g}
\end{array}\right], \\
\bar{B}(q) & =\frac{1}{r}\left[\begin{array}{cc}
1 & 1 \\
L & -L
\end{array}\right], \\
V_{m} & =\left[\begin{array}{l}
v \\
w
\end{array}\right], \\
\tau & =\left[\begin{array}{l}
\tau_{r} \\
\tau_{l}
\end{array}\right] .
\end{aligned}
$$

The expressions of linear and angular velocities of the mobile robot, $(v, w)$, depend on the left and right linear velocities of the motors. They are expressed by the following equations:

$$
\begin{gathered}
v=\frac{v_{r}+v_{l}}{2}, \\
w=\frac{v_{r}-v_{l}}{2 L} .
\end{gathered}
$$

\section{Design of Robot Controllers}

In this work, we consider the kinematic and dynamic behavior of the robot. The purpose of the control design is to allow the robot to follow the virtual robot. The latter represents the reference robot and provides the desired path defined by the following vector: $q_{d}(t)=\left[\begin{array}{lll}x_{d} & y_{d} & \theta_{d}\end{array}\right]^{T}$.

The architecture of the control scheme of the robot, includes six blocks, as shown in Figure 4. The first block generates the desired states, whereas the second block transforms the error from the local frame into the general frame. The third and fourth blocks are reserved, respectively, for kinetic and dynamic controllers. The fifth and sixth blocks, respectively, describe the behavior of the kinematic and dynamic models of the robot.

3.1. Fuzzy Kinematic Controller. In this section, we are interested in the search for a T-S type fuzzy controller, which guarantees the convergence of the kinematic errors towards zero in the local coordinate system and allows the robot to follow the desired path.

Based on the T-S fuzzy model (14), the rules for the local controllers are given by the following expressions:

$$
\begin{aligned}
& \text { if } e_{3} \text { is } \mu_{1}, \quad \text { then } V_{m}=\left[v=K_{1} e_{1}+v_{d} \omega=K_{3} e_{3}+\frac{v_{d} e_{2}}{e_{3}}+\omega_{d}\right]^{T}, \\
& \text { if } e_{3} \text { is } \mu_{2} \text {, then } V_{m}=\left[v=K_{1} e_{1}+v_{d} \omega=K_{3} e_{3}+\frac{v_{d} e_{2}}{e_{3}}+\omega_{d}\right]^{T}, \\
& \text { if } e_{3} \text { is } \mu_{3} \text {, then } V_{m}=\left[v=K_{1} e_{1}+v_{d} \omega=K_{3} e_{3}+\frac{v_{d} e_{2}}{e_{3}}+\omega_{d}\right]^{T} .
\end{aligned}
$$

The global T-S fuzzy controller is given by the following equation:

$$
\begin{aligned}
& \begin{array}{l}
V_{m}=\left[v_{c}=\sum_{i=1}^{3} \mu_{i} \nu_{i}=K_{1} e_{1}+\mu_{1} \nu \omega_{c}=\sum_{i=1}^{3} \mu_{i} \omega_{i}\right. \\
\left.\quad=\omega_{d}+K_{3} e_{3}+\mu_{1} v_{d} e_{2}+\left(\mu_{2}-\mu_{3}\right) \frac{v_{d} e_{2}}{e_{3}}\right]^{T} .
\end{array} \\
& \text { If } e_{3}=0 \text {, then } \mu_{1}=1 \text { and } \mu_{2}=\mu_{3}=0 \text {, so }
\end{aligned}
$$




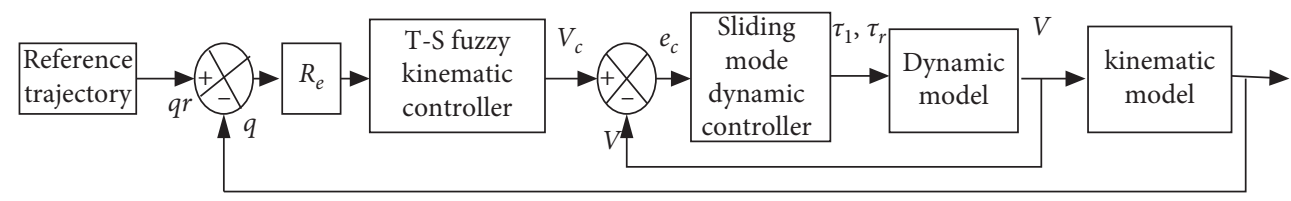

FIGURE 4: Architecture of the robot controller.

$$
\begin{aligned}
V_{m}= & {\left[\nu_{c}=\sum_{i=1}^{3} \mu_{i} \nu_{i}=K_{1} e_{1}+\mu_{1} \nu \omega_{c}\right.} \\
& \left.=\sum_{i=1}^{3} \mu_{i} \omega_{i}=\omega_{d}+K_{3} e_{3}+\mu_{1} \nu_{d} e_{2}\right]^{T} .
\end{aligned}
$$

3.1.1. Stability Analysis. To check the stability of the robot, we use Lyapunov's theory. However, we choose the following Lyapunov candidate function:

$$
V=\frac{1}{2} e_{1}^{2}+\frac{1}{2} e_{2}^{2}+\frac{1}{2} e_{3}^{2}
$$

The derivative of Lyapunov function is as follows. So,

$$
\begin{aligned}
\dot{V}= & \dot{e}_{1} e_{1}+\dot{e}_{2} e_{2}+\dot{e}_{3} e_{3}, \\
\dot{V}= & \left(\mu_{1}\left(\omega e_{2}-v+v_{d}\right)+\mu_{2}\left(\omega e_{2}-v\right)+\mu_{3}\left(\omega e_{2}-v\right)\right) e_{1} \\
& +\left(\mu_{1}\left(-\omega e_{1}+v_{d} e_{3}\right)+\mu_{2}\left(-\omega e_{1}+v_{d}\right)+\mu_{3}\left(-\omega e_{1}-v_{d}\right)\right) e_{2} \\
& +\left(\mu_{1}\left(\omega_{d}-\omega\right)+\mu_{2}\left(\omega_{d}-\omega\right)+\mu_{3}\left(\omega_{d}-\omega\right)\right) e_{3} .
\end{aligned}
$$

So,

$\dot{V}=\left(\left(-v+\mu_{1} v_{d}\right)\right) e_{1}+\left(\mu_{1} v_{d} e_{2}+\omega_{d}+\left(\mu_{2}-\mu_{3}\right) \frac{v_{d} e_{2}}{e_{3}}-\omega\right) e_{3}$.

If we choose the following linear and angular velocities,

$$
\begin{aligned}
& v_{c}=K_{1} e_{1}+\mu_{1} v_{d}, \\
& \omega_{c}=\sum_{i=1}^{3} \mu_{i} \omega_{i}=\omega_{d}+K_{3} e_{3}+\mu_{1} v_{d} e_{2}+\left(\mu_{2}-\mu_{3}\right) \frac{v_{d} e_{2}}{e_{3}} .
\end{aligned}
$$

Equation (26) becomes

$$
\dot{V}=-K_{1} e_{1}^{2}-K_{3} e_{3}^{2} \leq 0, \quad \text { if } e_{3} \neq 0 .
$$

If $e_{3}=0$, then $\mu_{1}=1$ and $\mu_{2}=\mu_{3}=0$. So, $\nu_{c}=K_{1} e_{1}+v_{d}$ and $\omega_{c}=\sum_{i=1}^{3} \mu_{i} \omega_{i}=\omega_{d}+K_{3} e_{3}+v_{d} e_{2}$.

Also,

$$
\dot{V}=-K_{1} e_{1}^{2}-K_{3} e_{3}^{2} \leq 0 .
$$

The derivative of the Lyapunov function is negative and the stability of the system is guarantee.

3.2. Dynamic Controller Based on Sliding Mode. In this section, we are interested in the development of a controller, which guarantees the convergence of the posture error $q_{e}$ towards zero for any arbitrary reference trajectory. However, we have developed a controller based on the sliding mode approach because the latter is considered a robust approach $[19,20]$. In this case, we define two sliding surfaces. The first surface depends on linear velocity, while the second uses angular velocity, $S=\left[\begin{array}{ll}s_{v} & s_{w}\end{array}\right]^{T}$ :

$$
S(t)=\left[\begin{array}{c}
S_{v} \\
S_{w}
\end{array}\right]=e_{e}(t)+K \int_{0}^{t} e_{e}(\delta) \mathrm{d} \delta
$$

where $s_{v}$ and $s_{w}$ are given, respectively, by equations (31) and (32), $e_{e}=\left(V_{c}-V_{m}\right)=\left[\begin{array}{ll}e_{v} & e_{w}\end{array}\right]^{T}$. With $e_{v}=v_{c}-v$ and $e_{w}=w_{c}-w$

$$
\begin{aligned}
& S_{v}(t)=e_{v}(t)+k_{v} \int_{0}^{t} e_{v}(\delta) \mathrm{d} \delta, \\
& S_{w}(t)=e_{w v}(t)+k_{w} \int_{0}^{t} e_{w}(\delta) \mathrm{d} \delta .
\end{aligned}
$$

However, the derivatives of the sliding surfaces $s_{v}(t)$ and $s_{w}(t)$ are given by the following expressions:

$$
\begin{gathered}
\dot{S}_{v}(t)=\dot{e}_{v}(t)+k_{v} e_{v}(t), \\
\dot{S}_{w}(t)=\dot{e}_{w}(t)+k_{w} e_{w}(t) .
\end{gathered}
$$

The dynamic motion of the robot is described by equation (7) which can be transformed as

$$
\dot{V}_{m}=(\bar{M}(q))^{-1} \bar{B}(q) \tau \text {. }
$$

Equation (34) can be written as

$$
\dot{V}_{m}=\widetilde{B} \tau
$$

where $\widetilde{B}=(\bar{M})^{-1} \bar{B}$

Based on the sliding mode theory, the controller includes two terms which are known as equivalent control law and switching control. The global control law is expressed as

$$
u=\tau=u_{\mathrm{eq}}+u_{s}=\tau_{\mathrm{eq}}+\tau_{s} .
$$

The equivalent control law $u_{\text {eq }}$ is computed by recognizing that $\dot{S}=0$ which is a necessary condition for the state trajectory to stay in the sliding surface $[19,20]$. The derivative of the sliding surface is

$$
\dot{S}(t)=\dot{e}_{e}(t)+K e_{e}(t)
$$

with $\dot{e}_{e}=\left(\dot{V}_{c}-\dot{V}_{m}\right)$ and $e_{e}=\left(V_{c}-V_{m}\right)$.

Thus, substituting (35) for (37), we obtain

$$
\dot{S}(t)=\dot{V}_{c}-\widetilde{B} \tau+K e_{e}(t)=\left[\begin{array}{l}
0 \\
0
\end{array}\right],
$$


with

$$
\begin{aligned}
\bar{M}^{-1} & =\frac{1}{m J_{g}}\left[\begin{array}{cc}
J_{g} & 0 \\
0 & m
\end{array}\right], \\
\widetilde{B} & =\bar{M}^{-1} \bar{B}=\frac{1}{r m J_{g}}\left[\begin{array}{cc}
J_{g} & J_{g} \\
m L & m L
\end{array}\right], \\
\widetilde{B}^{-1} & =\frac{r}{2 L}\left[\begin{array}{ll}
m L & J_{g} \\
m L & -J_{g}
\end{array}\right], \\
\bar{B}^{-1} & =\frac{1}{2 L r}\left[\begin{array}{cc}
L & 1 \\
L & -1
\end{array}\right] .
\end{aligned}
$$

However, the equivalent control law $u_{\text {eq }}=\tau_{\text {eq }}$ can be computed as

$$
u_{\mathrm{eq}}=\tau_{\mathrm{eq}}=\widetilde{B}^{-1} \dot{V}_{c}+\widetilde{B}^{-1} K e_{e}(t),
$$

with

$$
\dot{V}_{c}=\left[\begin{array}{c}
\dot{v}_{c}=K_{1} \dot{e}_{1}+\mu_{1} \dot{v}_{d} \\
\dot{\omega}_{c}=\dot{\omega}_{d}+K_{3} \dot{e}_{3}+\mu_{1}\left(\dot{v}_{d} e_{2}+v_{d} \dot{e}_{2}\right)+\left(\mu_{2}-\mu_{3}\right) \frac{\left(\dot{v}_{d} e_{2}+v_{d} \dot{e}_{2}\right) e_{3}-v_{d} e_{2} \dot{e}_{3}}{e_{3}^{2}}
\end{array}\right] .
$$

So,

$$
\begin{aligned}
& {\left[\begin{array}{c}
u_{\mathrm{eqr}} \\
u_{\mathrm{eql}}
\end{array}\right]=\left[\begin{array}{cc}
\frac{r m}{2} & \frac{r J_{g}}{2 L} \\
\frac{r m}{2} & -\frac{r J_{g}}{2 L}
\end{array}\right]\left(\left[\begin{array}{c}
K_{1} \dot{e}_{1}+\mu_{1} \dot{v}_{d} \\
\dot{\omega}_{d}+K_{3} \dot{e}_{3}+\mu_{1}\left(\dot{v}_{d} e_{2}+v_{d} \dot{e}_{2}\right)+\left(\mu_{2}-\mu_{3}\right) \frac{\left(\dot{v}_{d} e_{2}+v_{d} \dot{e}_{2}\right) e_{3}-v_{d} e_{2} \dot{e}_{3}}{e_{3}}
\end{array}\right]+\left[\begin{array}{l}
\lambda e_{v} \\
\lambda e_{w}
\end{array}\right]\right) \text {, }} \\
& {\left[\begin{array}{c}
u_{\mathrm{eqr}} \\
u_{\mathrm{eql}}
\end{array}\right]=\left[\begin{array}{cc}
\frac{r m}{2} & \frac{r J_{g}}{2 L} \\
\frac{r m}{2} & -\frac{r J_{g}}{2 L}
\end{array}\right]\left[\begin{array}{c}
K_{1} \dot{e}_{1}+\mu_{1} \dot{v}_{d}+\lambda e_{v} \\
\dot{\omega}_{d}+K_{3} \dot{e}_{3}+\mu_{1}\left(\dot{v}_{d} e_{2}+v_{d} \dot{e}_{2}\right)+\left(\mu_{2}-\mu_{3}\right)\left(\frac{\dot{v}_{d} e_{2}}{e_{3}}+\frac{v_{d} e_{2}}{e_{3}}-\frac{v_{d} e_{2} \dot{e}_{3}}{e_{3}^{2}}\right)+\lambda e_{w}
\end{array}\right],} \\
& {\left[\begin{array}{l}
u_{\text {eqr }} \\
u_{\text {eql }}
\end{array}\right]=\left[\begin{array}{l}
\frac{r m}{2}\left(K_{1} \dot{e}_{1}+\mu_{1} \dot{v}_{d}+\lambda e_{v}\right)+\frac{r J g}{2 L}\left(\dot{\omega}_{d}+K_{3} \dot{e}_{3}+\mu_{1}\left(\dot{v}_{d} e_{2}+v_{d} \dot{e}_{2}\right)+\left(\mu_{2}-\mu_{3}\right)\left(\frac{\left(\dot{v}_{d} e_{2}+v_{d} \dot{e}_{2}\right) e_{3}-v_{d} e_{2} \dot{e}_{3}}{e_{3}^{2}}\right)+\lambda e_{w}\right) \\
\frac{r m}{2}\left(\left(K_{1} \dot{e}_{1}+\mu_{1} \dot{v}_{d}+\lambda e_{v}\right)\right)-\frac{r J g}{2 L}\left(\dot{\omega}_{d}+K_{3} \dot{e}_{3}+\mu_{1}\left(\dot{v}_{d} e_{2}+v_{d} \dot{e}_{2}\right)+\left(\mu_{2}-\mu_{3}\right)\left(\frac{\left(\dot{v}_{d} e_{2}+v_{d} \dot{e}_{2}\right) e_{3}-v_{d} e_{2} \dot{e}_{3}}{e_{3}^{2}}\right)+\lambda e_{w}\right)
\end{array}\right] .}
\end{aligned}
$$

Finally, we obtain

$$
\begin{aligned}
& u_{\mathrm{eqr}}=\frac{r m}{2}\left(K_{1} \dot{e}_{1}+\mu_{1} \dot{v}_{d}+\lambda e_{v}\right)+\frac{r J g}{2 L}\left(\dot{\omega}_{d}+K_{3} \dot{e}_{3}+\mu_{1}\left(\dot{v}_{d} e_{2}+v_{d} \dot{e}_{2}\right)+\left(\mu_{2}-\mu_{3}\right)\left(\frac{\left(\dot{v}_{d} e_{2}+v_{d} \dot{e}_{2}\right) e_{3}-v_{d} e_{2} \dot{e}_{3}}{e_{3}^{2}}\right)+\lambda e_{w}\right) \\
& u_{\mathrm{eql}}=\frac{r m}{2}\left(\left(K_{1} \dot{e}_{1}+\mu_{1} \dot{v}_{d}+\lambda e_{v}\right)\right)-\frac{r J_{g}}{2 L}\left(\dot{\omega}_{d}+K_{3} \dot{e}_{3}+\mu_{1}\left(\dot{v}_{d} e_{2}+v_{d} \dot{e}_{2}\right)+\left(\mu_{2}-\mu_{3}\right)\left(\frac{\left(\dot{v}_{d} e_{2}+v_{d} \dot{e}_{2}\right) e_{3}-v_{d} e_{2} \dot{e}_{3}}{e_{3}^{2}}\right)+\lambda e_{w}\right) .
\end{aligned}
$$


The switching control term is generally chooses as $u_{s}=-\eta \operatorname{sign}(S)$, with $\eta>0$. This term can be canceled when the system reaches the sliding surface [20]. In this paper, the switching control law is chosen as follows:

$$
\tau_{s}=\left[\begin{array}{l}
-k_{s 1}\left(\operatorname{sgn}\left(s_{v}+s_{w}\right)\right) \\
-k_{s 1}\left(\operatorname{sgn}\left(s_{v}-s_{w}\right)\right)
\end{array}\right] .
$$

3.2.1. Reaching Condition and Stability Analysis. To verify the reaching condition, we need to just check the following condition. The Lyapunov candidate function is chosen as

$$
V=\frac{1}{2} S^{T} S
$$

The derivative can be expressed as

$$
\begin{aligned}
\dot{V} & =S^{T} \dot{S}, \\
\dot{V} & =S^{T}\left[\dot{V}_{c}-\widetilde{B} \tau+K e_{e}(t)\right], \\
\dot{V} & =S^{T}\left[\dot{V}_{c}-\widetilde{B}\left(\tau_{\text {eq }}+\tau_{s}\right)+K e_{e}(t)\right], \\
\dot{V} & =S^{T} \dot{S}=S^{T}\left[\dot{V}_{c}-\widetilde{B}\left(\tau_{\text {eq }}+\tau_{s}\right)+K e_{e}(t)\right] .
\end{aligned}
$$

Based on equation (40), $\dot{V}_{c}-\widetilde{B} \tau_{\text {eq }}+K e_{e}(t)=0$ So,

$\dot{V}=S^{T} \dot{S}=S^{T}\left[\begin{array}{cc}\frac{r m}{2} & \frac{r J_{g}}{2 L} \\ \frac{r m}{2} & -\frac{r J_{g}}{2 L}\end{array}\right]\left[\begin{array}{l}-k_{s}\left(\operatorname{sgn}\left(s_{v}+s_{w}\right)\right) \\ -k_{s}\left(\operatorname{sgn}\left(s_{v}-s_{w}\right)\right)\end{array}\right]$,

$\dot{V}=S^{T}\left[\begin{array}{c}-k_{s} \frac{r m}{2} \operatorname{sgn}\left(s_{v}+s_{w}\right)-k_{s} \frac{r J_{g}}{2 L} \operatorname{sgn}\left(s_{v}-s_{w}\right) \\ -k_{s} \frac{r m}{2} \operatorname{sgn}\left(s_{v}+s_{w}\right)+k_{s} \frac{r J_{g}}{2 L} \operatorname{sgn}\left(s_{v}-s_{w}\right)\end{array}\right]$,

$\dot{V}=\left[\begin{array}{ll}s_{v} & s_{w}\end{array}\right]\left[\begin{array}{l}-k_{s} \frac{r m}{2} \operatorname{sgn}\left(s_{v}+s_{w}\right)-k_{s} \frac{r J_{g}}{2 L} \operatorname{sgn}\left(s_{v}-s_{w}\right) \\ -k_{s} \frac{r m}{2} \operatorname{sgn}\left(s_{v}+s_{w}\right)+k_{s} \frac{r J_{g}}{2 L} \operatorname{sgn}\left(s_{v}-s_{w}\right)\end{array}\right]$,

$\dot{V}=\frac{k_{s} r m\left(s_{v}+s_{w}\right)}{2} \operatorname{sgn}\left(s_{v}+s_{w}\right)-\frac{k_{s} r J_{g}\left(s_{v}-s_{w}\right)}{2 L} \operatorname{sgn}\left(s_{v}-s_{w}\right)$,

$\dot{V}=-\frac{k_{s} r m}{2}\left|\left(s_{v}+s_{w}\right)\right|-\frac{k_{s} r J_{g}}{2 L}\left|\left(s_{v}-s_{w}\right)\right|$.

We note $k_{1 s}=\left(k_{s} r m\right) / 2$ and $k_{2 s}=\left(k_{s} r J_{g}\right) / 2 L$. So,

$$
\dot{V}=-k_{s}\left|s_{v}+s_{w}\right|-k_{2 s}\left|s_{v}-s_{w}\right|<0 \text {. }
$$

\section{Simulation Results}

In this part, we present the simulation results of the nonholonomic wheeled mobile robot. The parameters of the robot

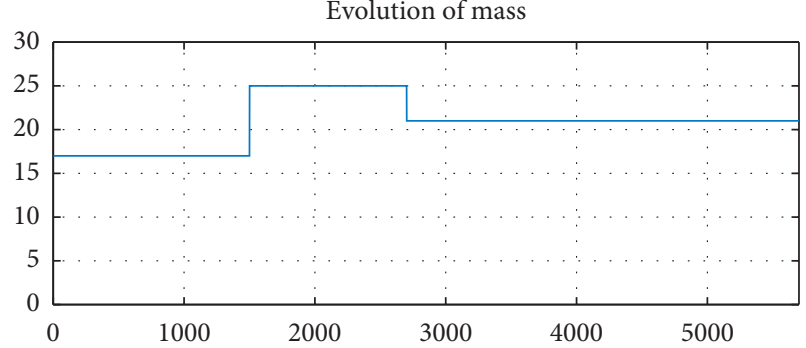

FIgURE 5: Evolution of mass of the robot.

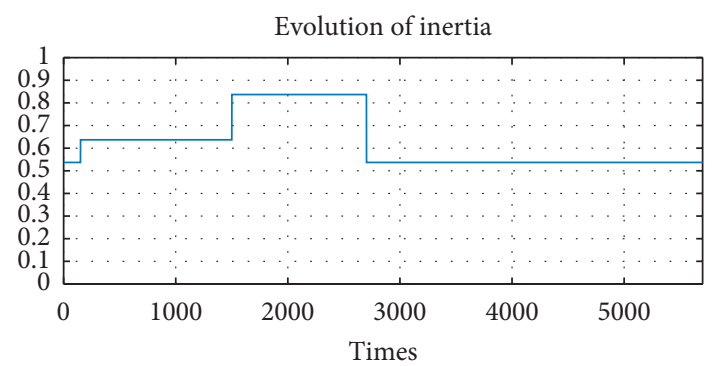

FIgURE 6: Evolution of inertia of the robot.

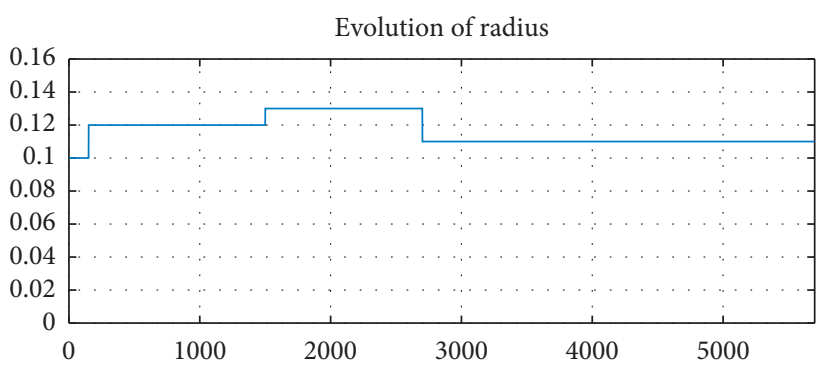

Figure 7: Evolution of radius of the robot.

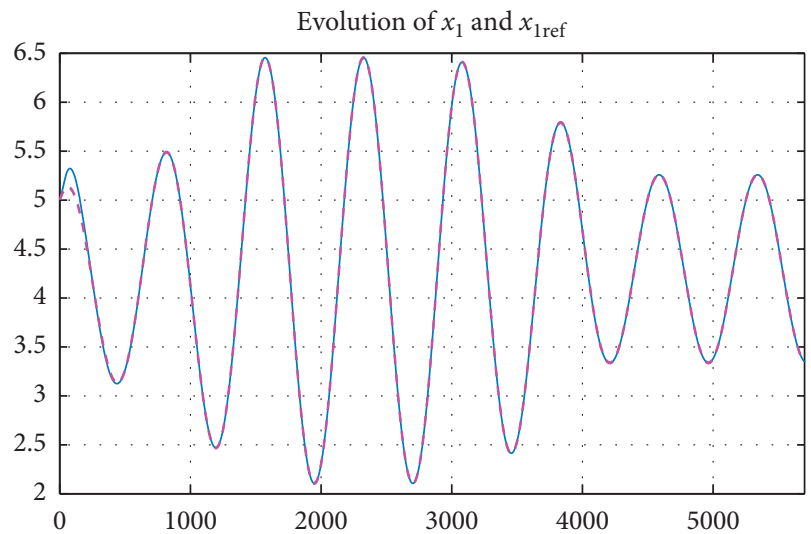

FIGURE 8: Evolution of the robot trajectory along the $x$-axis (blue denotes the trajectory and magenta denotes the reference trajectory).

(see Figure 1) are $m=17 \mathrm{~kg}, J=0.537 \mathrm{~kg} \cdot \mathrm{m}^{2}, L=0.24 \mathrm{~m}$, and $r=0.1 \mathrm{~m}$ and initial position [ $\left.\begin{array}{lll}x_{0} & y_{o} & \theta\end{array}\right]=\left[\begin{array}{lll}5 & 5 & \pi / 12\end{array}\right]$. The reference speed has been chosen as the variable as shown by the following equation: 


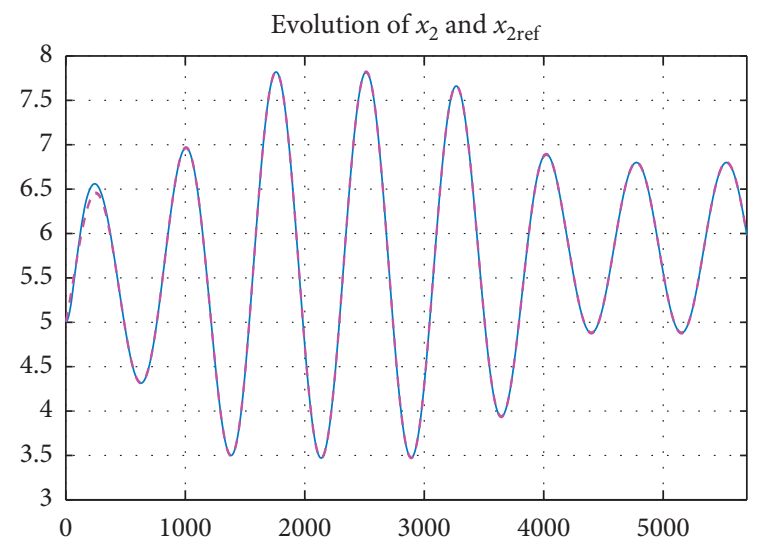

Figure 9: Evolution of the robot trajectory along the $y$-axis (blue denotes the trajectory and magenta denotes the reference trajectory).

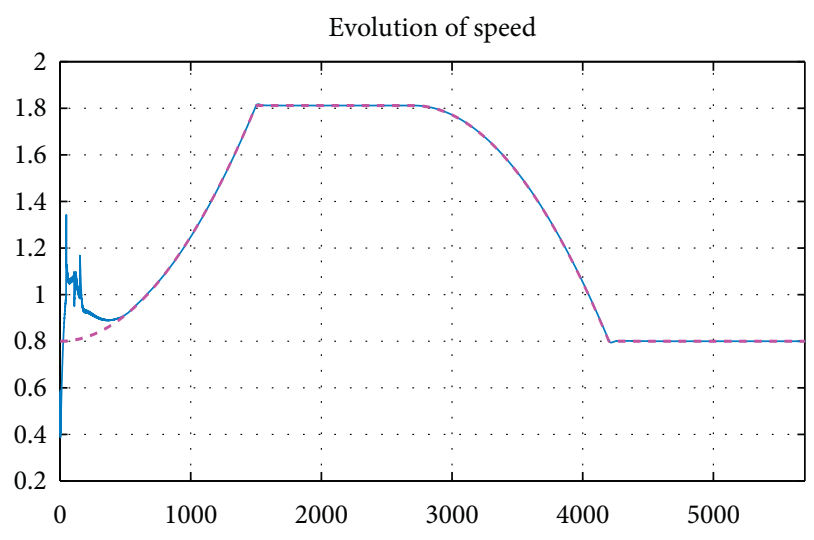

Figure 10: Evolution of linear speed $v$.

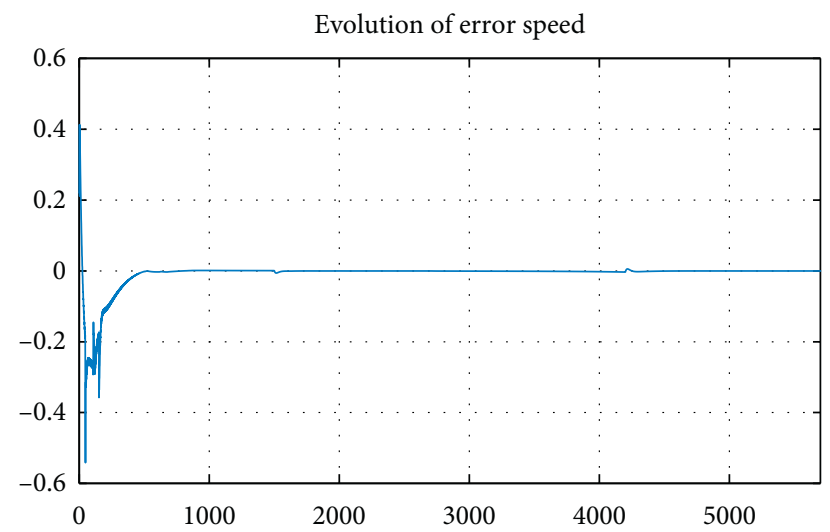

FIGURE 11: Evolution of the error of the linear speed $e v=v_{r}-v$.

$$
\begin{cases}v_{r}=v_{r o}+910^{-7} t, & \text { for } t \leq 15 \mathrm{~s}, \\ v_{r}=v_{r}, & \text { for } 15 \leq t \leq 27 \mathrm{~s}, \\ v_{r}=v_{r}-910^{-7} t, & \text { for } t>27 \mathrm{~s} \text { and } v_{r} \geq 0.8\end{cases}
$$

The initial speeds have been chosen as $v_{r o}=0.8$, $v_{d}=0.78$, and $v_{g}=0.38$.
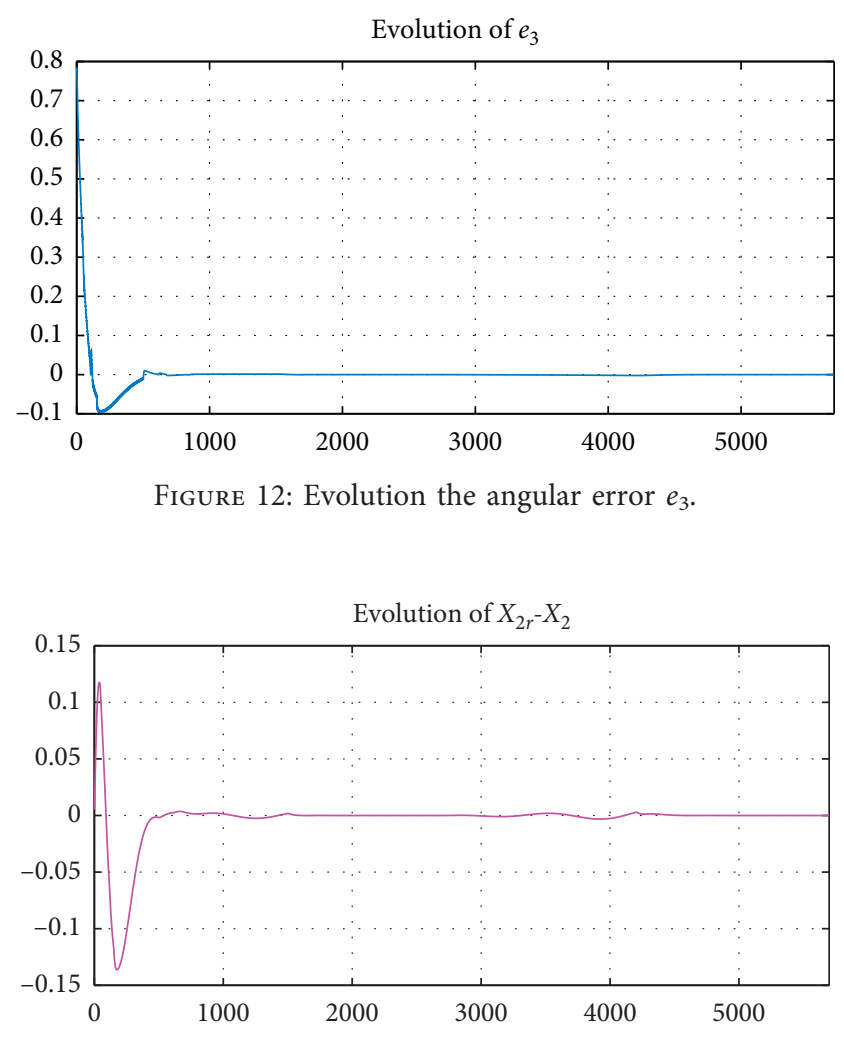

FIgURE 13: Evolution of the error $x_{r 2}-x_{2}$.

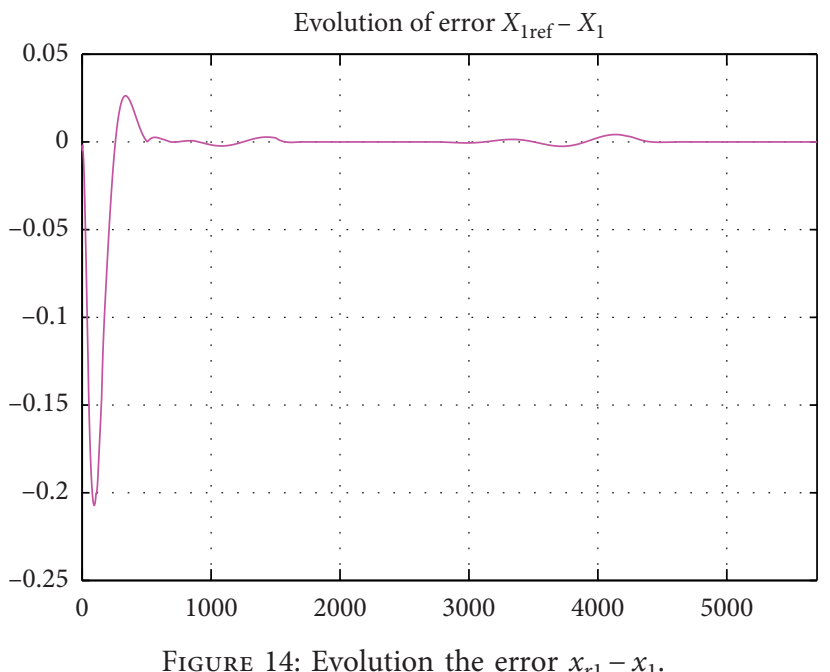

Figures 5-7 show, respectively, the evolution in time of mass, inertia, and radius of the robot.

Figures 8 and 9 show, respectively, the evolution in time of the robot along the $x$ - and $y$-axis (the trajectories of robot and its reference).

Figures 10 and 11 show, respectively, the behavior evolution of linear speed $v$ and the error speed $e v=v_{r}-v$.

Figures 8-10 show that all the signals follow quickly the reference. The curves of the reference signals and the responses overlap in a very short time after start-up.

Figure 12 shows the evolution the angular error $e_{3}$. 

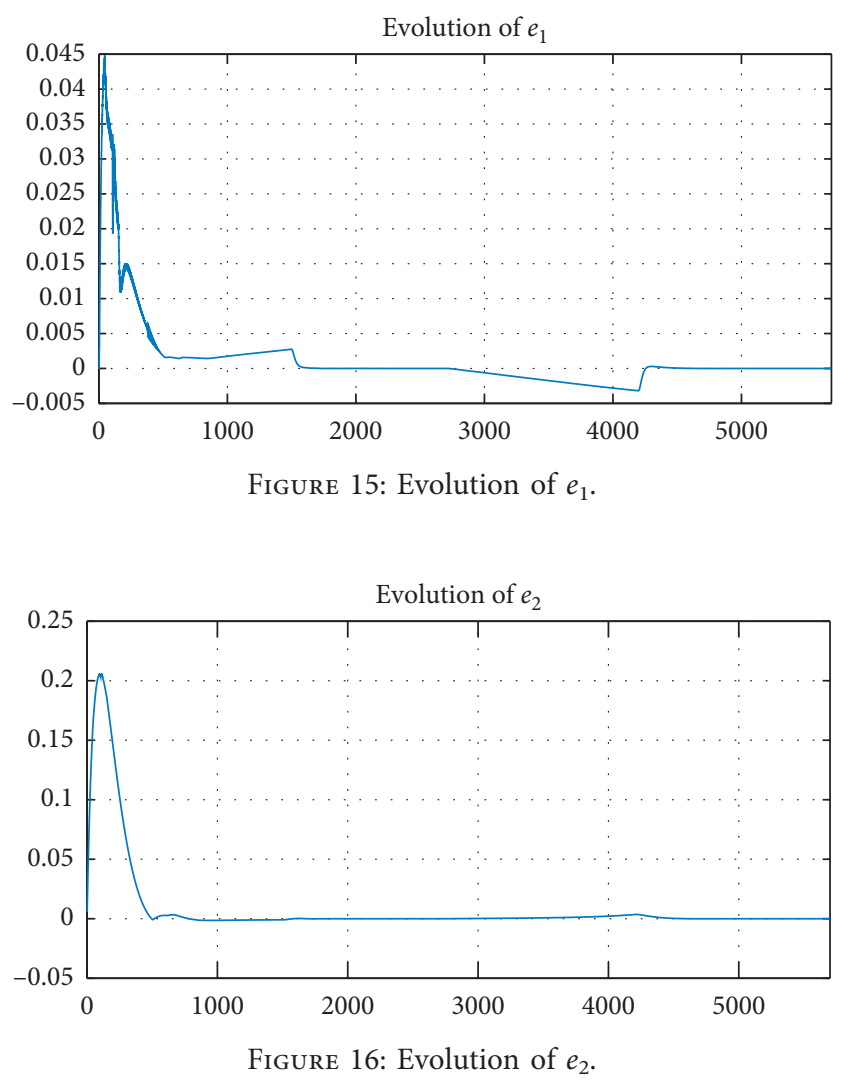

Figures 13 and 14 show, respectively, the behavior evolution of errors $x_{2 \mathrm{ref}}-x_{2}$ and $x_{1 \mathrm{ref}}-x_{1}$.

Figures 15 and 16 show, respectively, the behavior evolution of errors $e_{1}$ and $e_{2}$.

Figures 12-16 show that all the errors rapidly tend towards zero. This clearly shows that the system converges towards the desired trajectories in a very short time. This proves the effectiveness of the proposed command.

\section{Conclusion}

In this paper, we have presented a scheme to control a nonholonomic robot. We have proposed two controllers. The first concerns the kinematic behavior, while the second relates to the dynamic behavior of the mobile robot. To overcome the nonlinearities present in the model, we have used a Takagi-Sugeno fuzzy system for the kinematic controller. For the second controller, we have used the sliding mode technique, which is known as a robust technique. The proposed sliding surface has the same structure as the proportional integral controller. The Lyapunov approach has been used to prove stability of the system. The results presented in Section 4 show the efficiency of the proposed control laws.

\section{Data Availability}

There is no specific data used to support the conclusions of the study. All the necessary information exists in the paper.

\section{Conflicts of Interest}

The author declares that there are no conflicts of interest.

\section{References}

[1] T. Fukao, H. Nakagawa, and N. Adachi, "Adaptive tracking control of a nonholonomic mobile robot," IEEE Transactions on Robotics and Automation, vol. 16, no. 5, pp. 609-615, 2000.

[2] G. Zidani, D. Said, and L. Chrifi-Alaoui, A. Benmakhlouf and S. Chaouch, Back- stepping controller for a wheeled mobile robot," in Proceedings of the 4th International Conference on Systems and Control (ICSC'15), Sousse,Tunisia, April 2015.

[3] M. Egerstedt, X. Hu, and A. Stotsky, "Control of mobile platforms using a virtual vehicle approach," IEEE Transactions on Automatic Control, vol. 46, no. 11, pp. 1777-1782, 2001.

[4] J. Yang and J. kim, "Sliding mode motion control of nonholonomic mobile robots," IEEE Control Systems, vol. 19, no. 2, pp. 15-23, 1999.

[5] J.-K. Lee, J.-B. Park, and Y.-H. Choi, "Tracking control of nonholonomic wheeled mobile robot based on new sliding surface with approach angle," in Proceedings of the 3rd IFAC Symposium on Telematics Applications The International Federation of Automatic Control, Seoul, Republic of Korea, November 2013.

[6] M. A. Moqbel Obaid, A. R. Husain, and A. A. Mohammed Alkubati, "Robust backstepping tracking control of mobile robot based on nonlinear disturbance observer," International Journal of Electrical and Computer Engineering (IJECE), vol. 6, no. 2, pp. 901-908, 2016.

[7] A. V. Topalov, J.-H. Kim, and T. P. Proychev, "Fuzzy-net control of non-holonomic mobile robot using evolutionary feedback-error-learning," Robotics and Autonomous Systems, vol. 23, no. 3, pp. 187-200, 1998.

[8] R.-J. Chia-Ming Liu and C.-M. Liu, "Design of dynamic Petri recurrent fuzzy neural network and its application to pathtracking control of nonholonomic mobile robot," IEEE Transactions on Industrial Electronics, vol. 56, no. 7, pp. 2667-2683, 2009.

[9] A. Bessas, B. Atallah, and F. Boudjema, "Integral sliding mode control for trajectory tracking of wheeled mobile robot in presence of uncertainties," Journal of Control Science and Engineering, vol. 2016, Article ID 7915375, 10 pages, 2016.

[10] R. Fierro and F. L. Lewis, "Control of a nonholomic mobile robot: backstepping kinematics into dynamics," Journal of Robotic Systems, vol. 14, no. 3, pp. 149-163, 1997.

[11] E.-H. Guechi, J. Lauber, M. Dambrine, G. Klancar, and S. Blažic, "PDC control design for non-holonomic wheeled mobile robots with delayed outputs," Journal of Intelligent and Robotic Systems, vol. 60, no. 3, pp. 395-414, 2010.

[12] Y. Hung-Hsiu and H.-K. Huang, "Experimental verification for adaptive tracking control of a nonholonomic mobile robot," in Proceedings of the 33rd Annual Conference of the IEEE Industrial Electronics Society (IECON), Taipei, Taiwan, November 2007.

[13] J. K. Lee, Y. H. Choi, Y. H. Choi, and J. B. Park, Sliding mode tracking control of mobile robots with approach angle in cartesian coordinates," International Journal of Control, Automation and Systems, vol. 13, no. 3, pp. 718-724, 2015.

[14] A. Nikranjbar, A. Masoud Haidari, and B. Ali Asghar Atai, "Adaptive sliding mode tracking control of mobile robot in dynamic environment using artificial potential fields," Journal of Computer and Robotics, vol. 11, no. 1, pp. 1-14, 2018. 
[15] K. L. Besseghieur, R. Trębiński, W. Kaczmarek, and J. Panasiuk, "Trajectory tracking control for a nonholonomic mobile robot under ROS," Journal of Physics Conference Series, vol. 1016, Article ID 012008, 2018.

[16] J. Craig, Introduction to Robotics: Mechanics and Control, Addison-Wesley, Harlow, England, $1 \mathrm{em}$ plus $0.5 \mathrm{em}$ minus $0.4 \mathrm{em}, 1989$.

[17] M. K. Bugeja, S. G. Fabri, and L. Camilleri, "Dual adaptive dynamic control of mobile robots using neural networks," IEEE Transactions on Systems, Man, and Cybernetics, Part B (Cybernetics), vol. 39, no. 1, pp. 129-141, 2009.

[18] Y. Morère, "Mise en oeuvre de lois de commande pour les modèles flous de type TakagiSugeno," Université de Valenciennes et du Hainaut-Cambrésis, Valenciennes, France, Thèse de doctorat, 2001.

[19] H. Abid, C. Mohamed, and T. Ahmed, "An indirect adaptive fuzzy sliding mode controller for a class of SISO non-linear systems," International Journal of Modelling, Identification and Control, vol. 4, no. 2, 2008.

[20] Utkin, "Variable structure systems with sliding mode," IEEE Transactions on Automatic Control, vol. 22, no. 2, pp. 212-222, 1997. 